\title{
Brahmi (Bacopa monnieri Linn) in the treatment of dementias - a pilot study
}

\author{
Authors: Mohan Mishra, ${ }^{\text {A }}$ Ajay Kumar Mishra ${ }^{\text {B }}$ and Udbhatt Mishra ${ }^{B}$
}

\section{Aims}

To determine if Brahmi (Bacopa monnieri Linn), a herb used traditionally in India as a memory-enhancer, could be used in various forms of dementia.

\section{Methods}

Twelve patients of either sex, aged 18 years or more, entered the study. They all completed the study. Inclusion criteria: all grades of dementia; patients suffering from other coexisting diseases too, but in a stable condition, were included. Exclusion criteria: type 1 diabetes mellitus and pregnancy. The study was conducted between June 2015 and May 2016.

Every patient was administered $250 \mathrm{mg}$ of Brahmi (Himalaya) twice daily for 3 months. The Global Deterioration Scale (GDS) was determined at the beginning of the study and again at the end of 3 months.

\section{Results}

All the 12 patients showed a positive response. The GDS was: mean (standard deviation (SD)) 4.42 (1.38) before the start of treatment and mean (SD) 1.92 (1.31) at the end of 3 months. The fall was statistically highly significant: $p<0.001$. There were no significant adverse effects in any patient.

\section{Conclusion}

Brahmi is an effective drug for the treatment of dementias. This pilot study should open up ways for launching more definitive studies and provide a framework for work in the future.

\section{Conflict of interest statement}

None declared.

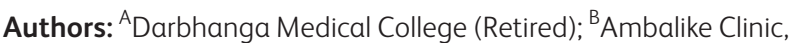
Darbhanga, India 